Annales Academiæ Scientiarum Fennicæ

Mathematica

Volumen 35, 2010, 405-420

\title{
APPROXIMATE IDENTITIES AND YOUNG TYPE INEQUALITIES IN VARIABLE LEBESGUE-ORLICZ SPACES $L^{p(\cdot)}(\log L)^{q(\cdot)}$
}

\section{Fumi-Yuki Maeda, Yoshihiro Mizuta and Takao Ohno}

4-24 Furue-higashi-machi, Nishi-ku, Hiroshima 733-0872, Japan; fymaeda@h6.dion.ne.jp

Hiroshima University, Graduate School of Science, Department of Mathematics Higashi-Hiroshima 739-8521, Japan; yomizuta@hiroshima-u.ac.jp Hiroshima National College of Maritime Technology, General Arts Higashino Oosakikamijima Toyotagun 725-0231, Japan; ohno@hiroshima-cmt.ac.jp

Abstract. Our aim in this paper is to deal with approximate identities in generalized Lebesgue spaces $L^{p(\cdot)}(\log L)^{q(\cdot)}\left(\mathbf{R}^{n}\right)$. As a related topic, we also study Young type inequalities for convolution with respect to norms in such spaces.

\section{Introduction}

Following Cruz-Uribe and Fiorenza [2], we consider two variable exponents $p(\cdot)$ : $\mathbf{R}^{n} \rightarrow[1, \infty)$ and $q(\cdot): \mathbf{R}^{n} \rightarrow \mathbf{R}$, which are continuous functions. Letting $\Phi_{p(\cdot), q(\cdot)}(x, t)$ $=t^{p(x)}\left(\log \left(c_{0}+t\right)\right)^{q(x)}$, we define the space $L^{p(\cdot)}(\log L)^{q(\cdot)}(\Omega)$ of all measurable functions $f$ on an open set $\Omega$ such that

$$
\int_{\Omega} \Phi_{p(\cdot), q(\cdot)}\left(y, \frac{|f(y)|}{\lambda}\right) d y<\infty
$$

for some $\lambda>0$; here we assume

$(\Phi) \Phi_{p(\cdot), q(\cdot)}(x, \cdot)$ is convex on $[0, \infty)$ for every fixed $x \in \mathbf{R}^{n}$.

Note that $(\Phi)$ holds for some $c_{0} \geq e$ if and only if there is a positive constant $K$ such that

$$
K(p(x)-1)+q(x) \geq 0 \quad \text { for all } x \in \mathbf{R}^{n}
$$

(see Appendix). Further, we see from $(\Phi)$ that $t^{-1} \Phi_{p(\cdot), q(\cdot)}(x, t)$ is nondecreasing in $t$.

We define the norm

$$
\|f\|_{\Phi_{p(\cdot), q(\cdot), \Omega}}=\inf \left\{\lambda>0: \int_{\Omega} \Phi_{p(\cdot), q(\cdot)}\left(y, \frac{|f(y)|}{\lambda}\right) d y \leq 1\right\}
$$

for $f \in L^{p(\cdot)}(\log L)^{q(\cdot)}(\Omega)$. Note that $L^{p(\cdot)}(\log L)^{q(\cdot)}(\Omega)$ is a Musielak-Orlicz space [9]. Such spaces have been studied in $[2,8,10]$. In case $q(\cdot)=0$ on $\mathbf{R}^{n}, L^{p(\cdot)}(\log L)^{q(\cdot)}(\Omega)$ is denoted by $L^{p(\cdot)}(\Omega)([7])$.

We assume throughout the article that our variable exponents $p(\cdot)$ and $q(\cdot)$ are continuous functions on $\mathbf{R}^{n}$ satisfying:

$$
\text { (p1) } 1 \leq p_{-}:=\inf _{x \in \mathbf{R}^{n}} p(x) \leq \sup _{x \in \mathbf{R}^{n}} p(x)=: p_{+}<\infty
$$

doi:10.5186/aasfm.2010.3526

2000 Mathematics Subject Classification: Primary 46E30.

Key words: Lebesgue spaces of variable exponent, approximate identity, Young's inequality for convolution. 
(p2) $|p(x)-p(y)| \leq \frac{C}{\log (e+1 /|x-y|)} \quad$ whenever $x \in \mathbf{R}^{n}$ and $y \in \mathbf{R}^{n}$;

(p3) $|p(x)-p(y)| \leq \frac{C}{\log (e+|x|)} \quad$ whenever $|y| \geq|x| / 2$;

(q1) $-\infty<q_{-}:=\inf _{x \in \mathbf{R}^{n}} q(x) \leq \sup _{x \in \mathbf{R}^{n}} q(x)=: q_{+}<\infty$;

(q2) $|q(x)-q(y)| \leq \frac{C}{\log (e+\log (e+1 /|x-y|))} \quad$ whenever $x \in \mathbf{R}^{n}$ and $y \in \mathbf{R}^{n}$ for a positive constant $C$.

We choose $p_{0} \geq 1$ as follows: we take $p_{0}=p_{-}$if $t^{-p_{-}} \Phi_{p(\cdot), q(\cdot)}(x, t)$ is uniformly almost increasing in $t$; more precisely, if there exists $C>0$ such that $s^{-p_{-}} \Phi_{p(\cdot), q(\cdot)}(x, s) \leq$ $C t^{-p_{-}} \Phi_{p(\cdot), q(\cdot)}(x, t)$ whenever $0<s<t$ and $x \in \mathbf{R}^{n}$. Otherwise we choose $1 \leq p_{0}<$ $p_{-}$. Then note that $t^{-p_{0}} \Phi_{p(\cdot), q(\cdot)}(x, t)$ is uniformly almost increasing in $t$ in any case.

Let $\phi$ be an integrable function on $\mathbf{R}^{n}$. For each $t>0$, define the function $\phi_{t}$ by $\phi_{t}(x)=t^{-n} \phi(x / t)$. Note that by a change of variables, $\left\|\phi_{t}\right\|_{L^{1}, \mathbf{R}^{n}}=\|\phi\|_{L^{1}, \mathbf{R}^{n}}$. We say that the family $\left\{\phi_{t}\right\}$ is an approximate identity if $\int_{\mathbf{R}^{n}} \phi(x) d x=1$. Define the radial majorant of $\phi$ to be the function

$$
\hat{\phi}(x)=\sup _{|y| \geq|x|}|\phi(y)| .
$$

If $\hat{\phi}$ is integrable, we say that the family $\left\{\phi_{t}\right\}$ is of potential-type.

Cruz-Uribe and Fiorenza [1] proved the following result:

Theorem A. Let $\left\{\phi_{t}\right\}$ be an approximate identity. Suppose that either

(1) $\left\{\phi_{t}\right\}$ is of potential-type, or

(2) $\phi \in L^{\left(p_{-}\right)^{\prime}}\left(\mathbf{R}^{n}\right)$ and has compact support.

Then

$$
\sup _{0<t \leq 1}\left\|\phi_{t} * f\right\|_{L^{p(\cdot), \mathbf{R}^{n}}} \leq C\|f\|_{L^{p(\cdot), \mathbf{R}^{n}}}
$$

and

$$
\lim _{t \rightarrow+0}\left\|\phi_{t} * f-f\right\|_{L^{p(\cdot)}, \mathbf{R}^{n}}=0
$$

for all $f \in L^{p(\cdot)}\left(\mathbf{R}^{n}\right)$.

Our aim in this note is to extend their result to the space $L^{p(\cdot)}(\log L)^{q(\cdot)}(\Omega)$ of two variable exponents.

Theorem 1.1. Let $\left\{\phi_{t}\right\}$ be a potential-type approximate identity. If $f \in L^{p(\cdot)}(\log$ $L)^{q(\cdot)}\left(\mathbf{R}^{n}\right)$, then $\left\{\phi_{t} * f\right\}$ converges to $f$ in $L^{p(\cdot)}(\log L)^{q(\cdot)}\left(\mathbf{R}^{n}\right)$ :

$$
\lim _{t \rightarrow 0}\left\|\phi_{t} * f-f\right\|_{\Phi_{p(\cdot), q(\cdot)}, \mathbf{R}^{n}=0 .}
$$

Theorem 1.2. Let $\left\{\phi_{t}\right\}$ be an approximate identity. Suppose that $\phi \in L^{\left(p_{0}\right)^{\prime}}\left(\mathbf{R}^{n}\right)$ and has compact support. If $f \in L^{p(\cdot)}(\log L)^{q(\cdot)}\left(\mathbf{R}^{n}\right)$, then $\left\{\phi_{t} * f\right\}$ converges to $f$ in $L^{p(\cdot)}(\log L)^{q(\cdot)}\left(\mathbf{R}^{n}\right)$ :

$$
\lim _{t \rightarrow 0}\left\|\phi_{t} * f-f\right\|_{\Phi_{p(\cdot), q(\cdot)}, \mathbf{R}^{n}=0 .}
$$

We show by an example that the conditions on $\phi$ are necessary; see Remarks 3.5 and 3.6 below.

Finally, in Section 4, we give some Young type inequalities for convolution with respect to the norms in $L^{p(\cdot)}(\log L)^{q(\cdot)}\left(\mathbf{R}^{n}\right)$. 


\section{The case of potential-type}

Throughout this paper, let $C$ denote various positive constants independent of the variables in question.

Let us begin with the following result due to Stein [11].

Lemma 2.1. Let $1 \leq p<\infty$ and $\left\{\phi_{t}\right\}$ be a potential-type approximate identity. Then for every $f \in L^{p}\left(\mathbf{R}^{n}\right),\left\{\phi_{t} * f\right\}$ converges to $f$ in $L^{p}\left(\mathbf{R}^{n}\right)$.

We denote by $B(x, r)$ the open ball centered at $x \in \mathbf{R}^{n}$ and with radius $r>0$. For a measurable set $E$, we denote by $|E|$ the Lebesgue measure of $E$.

The following is due to Lemma 2.6 in [8].

Lemma 2.2. Let $f$ be a nonnegative measurable function on $\mathbf{R}^{n}$ with $\|f\|_{\Phi_{p(\cdot), q(\cdot)}, \mathbf{R}^{n}}$ $\leq 1$ such that $f(x) \geq 1$ or $f(x)=0$ for each $x \in \mathbf{R}^{n}$. Set

$$
J=J(x, r, f)=\frac{1}{|B(x, r)|} \int_{B(x, r)} f(y) d y
$$

and

$$
L=L(x, r, f)=\frac{1}{|B(x, r)|} \int_{B(x, r)} \Phi_{p(\cdot), q(\cdot)}(y, f(y)) d y
$$

Then

$$
J \leq C L^{1 / p(x)}\left(\log \left(c_{0}+L\right)\right)^{-q(x) / p(x)},
$$

where $C>0$ does not depend on $x, r, f$.

Further we need the following result.

Lemma 2.3. Let $f$ be a nonnegative measurable function on $\mathbf{R}^{n}$ such that $(1+$ $|y|)^{-n-1} \leq f(y) \leq 1$ or $f(y)=0$ for each $y \in \mathbf{R}^{n}$. Set

$$
J=J(x, r, f)=\frac{1}{|B(x, r)|} \int_{B(x, r)} f(y) d y
$$

and

$$
L=L(x, r, f)=\frac{1}{|B(x, r)|} \int_{B(x, r)} \Phi_{p(\cdot), q(\cdot)}(y, f(y)) d y .
$$

Then

$$
J \leq C\left\{L^{1 / p(x)}+(1+|x|)^{-n-1}\right\},
$$

where $C>0$ does not depend on $x, r, f$.

Proof. We have by Jensen's inequality

$$
\begin{aligned}
J \leq & \left(\frac{1}{|B(x, r)|} \int_{B(x, r)} f(y)^{p(x)} d y\right)^{1 / p(x)} \\
\leq & \left(\frac{1}{|B(x, r)|} \int_{B(x, r) \cap B(0,|x| / 2)} f(y)^{p(x)} d y\right)^{1 / p(x)} \\
& +\left(\frac{1}{|B(x, r)|} \int_{B(x, r) \backslash B(0,|x| / 2)} f(y)^{p(x)} d y\right)^{1 / p(x)} \\
= & J_{1}+J_{2} .
\end{aligned}
$$


We see from (p3) that

$$
J_{1} \leq C\left(\frac{1}{|B(x, r)|} \int_{B(x, r) \cap B(0,|x| / 2)} f(y)^{p(y)} d y\right)^{1 / p(x)} .
$$

Similarly, setting $E_{2}=\left\{y \in \mathbf{R}^{n}: f(y) \geq(1+|x|)^{-n-1}\right\}$, we see from (p3) that

$$
\begin{aligned}
J_{2} \leq & C\left(\frac{1}{|B(x, r)|} \int_{\{B(x, r) \backslash B(0,|x| / 2)\} \cap E_{2}} f(y)^{p(y)} d y\right)^{1 / p(x)} \\
& +\left(\frac{1}{|B(x, r)|} \int_{\{B(x, r) \backslash B(0,|x| / 2)\} \backslash E_{2}}(1+|x|)^{-p(x)(n+1)} d y\right)^{1 / p(x)} \\
\leq & C\left\{\left(\frac{1}{|B(x, r)|} \int_{B(x, r)} f(y)^{p(y)} d y\right)^{1 / p(x)}+(1+|x|)^{-(n+1)}\right\} .
\end{aligned}
$$

Since $f(y) \leq 1, f(y)^{p(y)} \leq C \Phi_{p(\cdot), q(\cdot)}(y, f(y))$. Hence, we have the required estimate.

By using Lemmas 2.2 and 2.3, we show the following theorem.

Theorem 2.4. If $\left\{\phi_{t}\right\}$ is of potential-type, then

$$
\left\|\phi_{t} * f\right\|_{\Phi_{p(\cdot), q(\cdot)}, \mathbf{R}^{n}} \leq C\|\hat{\phi}\|_{L^{1}, \mathbf{R}^{n}}\|f\|_{\Phi_{p(\cdot), q(\cdot)}, \mathbf{R}^{n}}
$$

for all $t>0$ and $f \in L^{p(\cdot)}(\log L)^{q(\cdot)}\left(\mathbf{R}^{n}\right)$.

Proof. Suppose $\|\hat{\phi}\|_{L^{1}, \mathbf{R}^{n}}=1$ and take a nonnegative measurable function $f$ on $\mathbf{R}^{n}$ such that $\|f\|_{\Phi_{p(\cdot), q(\cdot)}, \mathbf{R}^{n}} \leq 1$. Write

$$
\begin{aligned}
f & =f \chi_{\left\{y \in \mathbf{R}^{n}: f(y) \geq 1\right\}}+f \chi_{\left\{y \in \mathbf{R}^{n}:(1+|y|)^{-n-1} \leq f(y)<1\right\}}+f \chi_{\left\{y \in \mathbf{R}^{n}: f(y) \leq(1+|y|)^{-n-1}\right\}} \\
& =f_{1}+f_{2}+f_{3}
\end{aligned}
$$

where $\chi_{E}$ denotes the characteristic function of a measurable set $E \subset \mathbf{R}^{n}$.

Since $\hat{\phi}_{t}$ is a radial function, we write $\hat{\phi}_{t}(r)$ for $\hat{\phi}_{t}(x)$ when $|x|=r$. First note that

$$
\begin{aligned}
\left|\phi_{t} * f(x)\right| & \leq \int_{\mathbf{R}^{n}} \hat{\phi}_{t}(|x-y|) f_{1}(y) d y \\
& =\int_{0}^{\infty}\left(\frac{1}{|B(x, r)|} \int_{B(x, r)} f_{1}(y) d y\right)|B(x, r)| d\left(-\hat{\phi}_{t}(r)\right),
\end{aligned}
$$

so that Jensen's inequality and Lemma 2.2 yield

$$
\begin{aligned}
& \Phi_{p(\cdot), q(\cdot)}\left(x,\left|\phi_{t} * f_{1}(x)\right|\right) \\
& \leq \int_{0}^{\infty} \Phi_{p(\cdot), q(\cdot)}\left(x, \frac{1}{|B(x, r)|} \int_{B(x, r)} f_{1}(y) d y\right)|B(x, r)| d\left(-\hat{\phi}_{t}(r)\right) \\
& \leq C \int_{0}^{\infty}\left(\frac{1}{|B(x, r)|} \int_{B(x, r)} \Phi_{p(\cdot), q(\cdot)}\left(y, f_{1}(y)\right) d y\right)|B(x, r)| d\left(-\hat{\phi}_{t}(r)\right) \\
& =C\left(\hat{\phi}_{t} * g\right)(x),
\end{aligned}
$$


where $g(y)=\Phi_{p(\cdot), q(\cdot)}(y, f(y))$. The usual Young inequality for convolution gives

$$
\begin{aligned}
\int_{\mathbf{R}^{n}} \Phi_{p(\cdot), q(\cdot)}\left(x,\left|\phi_{t} * f_{1}(x)\right|\right) d x & \leq C \int_{\mathbf{R}^{n}}\left(\hat{\phi}_{t} * g\right)(x) d x \\
& \leq C\left\|\hat{\phi}_{t}\right\|_{L^{1}, \mathbf{R}^{n}}\|g\|_{L^{1}, \mathbf{R}^{n}} \leq C .
\end{aligned}
$$

Similarly, noting that $\frac{1}{|B(x, r)|} \int_{B(x, r)} f_{2}(y) d y \leq 1$ and applying Lemma 2.3, we derive the same result for $f_{2}$.

Finally, noting that $\left|\phi_{t} * f_{3}\right| \leq C\left\|\phi_{t}\right\|_{L^{1}, \mathbf{R}^{n}} \leq C$, we obtain

$$
\begin{aligned}
\int_{\mathbf{R}^{n}} \Phi_{p(\cdot), q(\cdot)}\left(x,\left|\phi_{t} * f_{3}(x)\right|\right) d x & \leq C \int_{\mathbf{R}^{n}}\left|\phi_{t} * f_{3}(x)\right| d x \\
& \leq C\left\|\phi_{t}\right\|_{L^{1}, \mathbf{R}^{n}}\left\|f_{3}\right\|_{L^{1}, \mathbf{R}^{n}} \leq C,
\end{aligned}
$$

as required.

We are now ready to prove Theorem 1.1.

Proof of Theorem 1.1. Given $\varepsilon>0$, we find a bounded function $g$ in $L^{p(\cdot)}(\log L)^{q(\cdot)}$ $\left(\mathbf{R}^{n}\right)$ with compact support such that $\|f-g\|_{\Phi_{p(\cdot), q(\cdot)}, \mathbf{R}^{n}}<\varepsilon$. By Theorem 2.4 we have

$$
\begin{aligned}
& \left\|\phi_{t} * f-f\right\|_{\Phi_{p(\cdot), q(\cdot)}, \mathbf{R}^{n}} \\
& \leq\left\|\phi_{t} *(f-g)\right\|_{\Phi_{p(\cdot), q(\cdot)}, \mathbf{R}^{n}}+\left\|\phi_{t} * g-g\right\|_{\Phi_{p(\cdot), q(\cdot)}, \mathbf{R}^{n}}+\|f-g\|_{\Phi_{p(\cdot), q(\cdot)}, \mathbf{R}^{n}} \\
& \leq C \varepsilon+\left\|\phi_{t} * g-g\right\|_{\Phi_{p(\cdot), q(\cdot)}, \mathbf{R}^{n}} .
\end{aligned}
$$

Since $\left|\phi_{t} * g\right| \leq\|g\|_{L^{\infty}, \mathbf{R}^{n}}$,

$$
\left\|\phi_{t} * g-g\right\|_{\Phi_{p(\cdot), q(\cdot)}, \mathbf{R}^{n}} \leq C^{\prime}\left\|\phi_{t} * g-g\right\|_{L^{1}, \mathbf{R}^{n}} \rightarrow 0
$$

by Lemma 2.1. (Here $C^{\prime}$ depends on $\|g\|_{L^{\infty}, \mathbf{R}^{n}}$.) Hence

$$
\limsup _{t \rightarrow 0}\left\|\phi_{t} * f-f\right\|_{\Phi_{p(\cdot), q(\cdot)}, \mathbf{R}^{n}} \leq C \varepsilon,
$$

which completes the proof.

As another application of Lemmas 2.2 and 2.3, we can prove the following result, which is an extension of [4, Theorem 1.5] and [8, Theorem 2.7] (see also [6]).

Let $M f$ be the Hardy-Littlewood maximal function of $f$.

Proposition 2.5. Suppose $p_{-}>1$. Then the operator $M$ is bounded from $L^{p(\cdot)}(\log L)^{q(\cdot)}\left(\mathbf{R}^{n}\right)$ to $L^{p(\cdot)}(\log L)^{q(\cdot)}\left(\mathbf{R}^{n}\right)$.

Proof. Let $f$ be a nonnegative measurable function on $\mathbf{R}^{n}$ such that $\|f\|_{\Phi_{p(\cdot), q(\cdot)}, \mathbf{R}^{n}}$ $\leq 1$ and write $f=f_{1}+f_{2}+f_{3}$ as in the proof of Theorem 2.4. Take $1<p_{1}<p_{-}$ and apply Lemmas 2.2 and 2.3 with $p(\cdot)$ and $q(\cdot)$ replaced by $p(\cdot) / p_{1}$ and $q(\cdot) / p_{1}$, respectively. Then

and

$$
\Phi_{p(\cdot), q(\cdot)}\left(x, M f_{1}(x)\right) \leq C\left[M g_{1}(x)\right]^{p_{1}}
$$

$$
\Phi_{p(\cdot), q(\cdot)}\left(x, M f_{2}(x)\right) \leq C\left\{\left[M g_{1}(x)\right]^{p_{1}}+(1+|x|)^{-n-1}\right\},
$$

where $g_{1}(y)=\Phi_{p(\cdot) / p_{1}, q(\cdot) / p_{1}}(y, f(y))$. As to $f_{3}$, we have

$$
\Phi_{p(\cdot), q(\cdot)}\left(x, M f_{3}(x)\right) \leq C\left[M f_{3}(x)\right]^{p_{1}} .
$$

Then the boundedness of the maximal operator in $L^{p_{1}}\left(\mathbf{R}^{n}\right)$ proves the proposition. 
Remark 2.6. If $p_{-}>1$, then the function $\Phi_{p(\cdot), q(\cdot)}$ is a proper $N$-function and our Proposition 2.5 implies that this function is of class $\mathscr{A}$ in the sense of Diening [5] (see [5, Lemma 3.2]). It would be an interesting problem to see whether "class $\mathscr{A}$ " is also a sufficient condition or not for the boundedness of $M$ on $L^{p(\cdot)}(\log L)^{q(\cdot)}\left(\mathbf{R}^{n}\right)$.

\section{The case of compact support}

We know the following result due to Zo [12]; see also [1, Theorem 2.2].

Lemma 3.1. Let $1 \leq p<\infty, 1 / p+1 / p^{\prime}=1$ and $\left\{\phi_{t}\right\}$ be an approximate identity. Suppose that $\phi \in L^{p^{\prime}}\left(\mathbf{R}^{n}\right)$ has compact support. Then for every $f \in L^{p}\left(\mathbf{R}^{n}\right)$, $\left\{\phi_{t} * f\right\}$ converges to $f$ pointwise almost everywhere.

Set

$$
\bar{p}(x)=p(x) / p_{0} \quad \text { and } \quad \bar{q}(x)=q(x) / p_{0} ;
$$

recall that $p_{0} \in\left[1, p_{-}\right]$is chosen such that $t^{-p_{0}} \Phi_{p(\cdot), q(\cdot)}(x, t)$ is uniformly almost increasing in $t$.

For a proof of Theorem 1.2, the following is a key lemma.

Lemma 3.2. Let $f$ be a nonnegative measurable function on $\mathbf{R}^{n}$ with $\|f\|_{\Phi_{p(\cdot), q(\cdot), \mathbf{R}^{n}}}$ $\leq 1$ such that $f(x) \geq 1$ or $f(x)=0$ for each $x \in \mathbf{R}^{n}$ and let $\phi$ have compact support in $B(0, R)$ with $\|\phi\|_{L^{\left(p_{0}\right)^{\prime}}, \mathbf{R}^{n}} \leq 1$. Set

$$
F=F(x, t, f)=\left|\phi_{t} * f(x)\right|
$$

and

$$
G=G(x, t, f)=\int_{\mathbf{R}^{n}}\left|\phi_{t}(x-y)\right| \Phi_{\bar{p}(\cdot), \bar{q}(\cdot)}(y, f(y)) d y .
$$

Then

$$
F \leq C G^{1 / \bar{p}(x)}\left(\log \left(c_{0}+G\right)\right)^{-\bar{q}(x) / \bar{p}(x)}
$$

for all $0<t \leq 1$, where $C>0$ depends on $R$.

Proof. Let $f$ be a nonnegative measurable function on $\mathbf{R}^{n}$ with $\|f\|_{\Phi_{p(\cdot), q(\cdot)}, \mathbf{R}^{n}} \leq 1$ such that $f(x) \geq 1$ or $f(x)=0$ for each $x \in \mathbf{R}^{n}$ and let $\phi$ have compact support in $B(0, R)$ with $\|\phi\|_{L^{\left(p_{0}\right)^{\prime}}, \mathbf{R}^{n}} \leq 1$. By Hölder's inequality, we have

$$
G \leq\left\|\phi_{t}\right\|_{L^{\left(p_{0}\right)^{\prime}}, \mathbf{R}^{n}}\left(\int_{\mathbf{R}^{n}} \Phi_{p(\cdot), q(\cdot)}(y, f(y)) d y\right)^{1 / p_{0}} \leq t^{-n / p_{0}} .
$$

First consider the case when $G \geq 1$. Since $G \leq t^{-n / p_{0}}$, for $y \in B(x, t R)$ we have by (p2)

$$
G^{-p(y)} \leq G^{-p(x)+C / \log \left(e+(t R)^{-1}\right)} \leq C G^{-p(x)}
$$

and by (q2)

$$
\left(\log \left(c_{0}+G\right)\right)^{q(y)} \leq C\left(\log \left(c_{0}+G\right)\right)^{q(x)} .
$$


Hence it follows from the choice of $p_{0}$ that

$$
\begin{aligned}
F \leq & G^{1 / \bar{p}(x)}\left(\log \left(c_{0}+G\right)\right)^{-\bar{q}(x) / \bar{p}(x)} \int_{\mathbf{R}^{n}}\left|\phi_{t}(x-y)\right| d y \\
& +C \int_{\mathbf{R}^{n}}\left|\phi_{t}(x-y)\right| f(y)\left\{\frac{f(y)}{G^{1 / \bar{p}(x)}\left(\log \left(c_{0}+G\right)\right)^{-\bar{q}(x) / \bar{p}(x)}}\right\}^{\bar{p}(y)-1} \\
& \cdot\left\{\frac{\log \left(c_{0}+f(y)\right)}{\log \left(c_{0}+G^{1 / \bar{p}(x)}\left(\log \left(c_{0}+G\right)\right)^{-\bar{q}(x) / \bar{p}(x)}\right)}\right\}^{\bar{q}(y)} d y \\
\leq & C G^{1 / \bar{p}(x)}\left(\log \left(c_{0}+G\right)\right)^{-\bar{q}(x) / \bar{p}(x)}
\end{aligned}
$$

(cf. the proof of $[8$, Lemma 2.6]).

In the case $G<1$, noting from the choice of $p_{0}$ that $f(y) \leq C \Phi_{\bar{p}(\cdot), \bar{q}(\cdot)}(y, f(y))$ for $y \in \mathbf{R}^{n}$, we find

$$
F \leq C G \leq C G^{1 / \bar{p}(x)} \leq C G^{1 / \bar{p}(x)}\left(\log \left(c_{0}+G\right)\right)^{-\bar{q}(x) / \bar{p}(x)} .
$$

Now the result follows.

Lemma 3.3. Suppose that $\|\phi\|_{L^{1}, \mathbf{R}^{n}} \leq 1$. Let $f$ be a nonnegative measurable function on $\mathbf{R}^{n}$ with $\|f\|_{\Phi_{p(\cdot), q(\cdot)}, \mathbf{R}^{n}} \leq 1$. Set

$$
I=I(x, t, f)=\int_{\left\{y \in \mathbf{R}^{n}:|y|>|x| / 2\right\}}\left|\phi_{t}(x-y)\right| f(y) d y
$$

and

$$
H=H(x, t, f)=\int_{\mathbf{R}^{n}}\left|\phi_{t}(x-y)\right| \Phi_{p(\cdot), q(\cdot)}(y, f(y)) d y .
$$

If $A>0$ and $H \leq H_{0}$, then

$$
I \leq C\left(H^{1 / p(x)}+|x|^{-A / p(x)}\right)
$$

for $|x|>1$ and $0<t \leq 1$, where $C>0$ depends on $A$ and $H_{0}$.

Proof. Suppose that $\|\phi\|_{L^{1}, \mathbf{R}^{n}} \leq 1$. Let $f$ be a nonnegative measurable function on $\mathbf{R}^{n}$ with $\|f\|_{\Phi_{p(\cdot), q(\cdot)}, \mathbf{R}^{n}} \leq 1$.

Let $|x|>1$. In the case $H_{0} \geq H \geq|x|^{-A}$ with $A>0$, we have by (p3)

$$
H^{-p(y)} \leq C H^{-p(x)-C / \log (e+|x|)} \leq C H^{-p(x)}
$$

for $|y| \geq|x| / 2$. Hence we find by $(\Phi)$

$$
\begin{aligned}
I \leq & C\left\{H^{1 / p(x)}+\int_{\left\{y \in \mathbf{R}^{n}:|y|>|x| / 2\right\}}\left|\phi_{t}(x-y)\right| f(y)\right. \\
& \left.\cdot\left\{\frac{f(y)}{H^{1 / p(x)}}\right\}^{p(y)-1}\left\{\frac{\log \left(c_{0}+f(y)\right)}{\log \left(c_{0}+H^{1 / p(x)}\right)}\right\}^{q(y)} d y\right\} \\
\leq & C H^{1 / p(x)} .
\end{aligned}
$$

Next note from (p3) that

$$
|x|^{p(y)} \leq|x|^{p(x)+C / \log (e+|x|)} \leq C|x|^{p(x)}
$$


for $|y| \geq|x| / 2$. Hence, when $H \leq|x|^{-A}$, we obtain by $(\Phi)$

$$
\begin{aligned}
I \leq & C\left\{|x|^{-A / p(x)}+\int_{\left\{y \in \mathbf{R}^{n}:|y|>|x| / 2\right\}}\left|\phi_{t}(x-y)\right| f(y)\right. \\
& \left.\cdot\left\{\frac{f(y)}{|x|^{-A / p(x)}}\right\}^{p(y)-1}\left\{\frac{\log \left(c_{0}+f(y)\right)}{\log \left(c_{0}+|x|^{-A / p(x)}\right)}\right\}^{q(y)} d y\right\} \\
\leq C|x|^{-A / p(x)}, &
\end{aligned}
$$

which completes the proof.

Theorem 3.4. Suppose that $\phi \in L^{\left(p_{0}\right)^{\prime}}\left(\mathbf{R}^{n}\right)$ has compact support in $B(0, R)$. Then

$$
\left\|\phi_{t} * f\right\|_{\Phi_{p(\cdot), q(\cdot)}, \mathbf{R}^{n}} \leq C\|\phi\|_{L^{\left(p_{0}\right)^{\prime}, \mathbf{R}^{n}}}\|f\|_{\Phi_{p(\cdot), q(\cdot)}, \mathbf{R}^{n}}
$$

for all $0<t \leq 1$ and $f \in L^{p(\cdot)}(\log L)^{q(\cdot)}\left(\mathbf{R}^{n}\right)$, where $C>0$ depends on $R$.

Proof. Let $f$ be a nonnegative measurable function on $\mathbf{R}^{n}$ such that $\|f\|_{\Phi_{p(\cdot), q(\cdot)}, \mathbf{R}^{n}}$ $\leq 1$ and let $\phi$ have compact support in $B(0, R)$ with $\|\phi\|_{L^{\left(p_{0}\right)^{\prime}}, \mathbf{R}^{n}} \leq 1$. Write

$$
f=f \chi_{\left\{y \in \mathbf{R}^{n}: f(y) \geq 1\right\}}+f \chi_{\left\{y \in \mathbf{R}^{n}: f(y)<1\right\}}=f_{1}+f_{2} .
$$

We have by Lemma 3.2,

$$
\left|\phi_{t} * f_{1}(x)\right| \leq C\left(\left|\phi_{t}\right| * g(x)\right)^{p_{0} / p(x)}\left(\log \left(c_{0}+\left|\phi_{t}\right| * g(x)\right)\right)^{-q(x) / p(x)},
$$

where $g(y)=\Phi_{\bar{p}(\cdot), \bar{q}(\cdot)}(y, f(y))=\Phi_{p(\cdot), q(\cdot)}(y, f(y))^{1 / p_{0}}$, so that

$$
\Phi_{p(\cdot), q(\cdot)}\left(x,\left|\phi_{t} * f_{1}(x)\right|\right) \leq C\left(\left|\phi_{t}\right| * g(x)\right)^{p_{0}} .
$$

Hence, since $g \in L^{p_{0}}\left(\mathbf{R}^{n}\right)$, the usual Young inequality for convolution gives

$$
\begin{aligned}
\int_{\mathbf{R}^{n}} \Phi_{p(\cdot), q(\cdot)}\left(x,\left|\phi_{t} * f_{1}(x)\right|\right) d x & \leq C \int_{\mathbf{R}^{n}}\left(\left|\phi_{t}\right| * g(x)\right)^{p_{0}} d x \\
& \leq C\left(\left\|\phi_{t}\right\|_{L^{1}, \mathbf{R}^{n}}\|g\|_{L^{p_{0}, \mathbf{R}^{n}}}\right)^{p_{0}} \leq C .
\end{aligned}
$$

Next we are concerned with $f_{2}$. Write

$$
f_{2}=f_{2} \chi_{B(0, R)}+f_{2} \chi_{\mathbf{R}^{n} \backslash B(0, R)}=f_{2}^{\prime}+f_{2}^{\prime \prime} .
$$

Since $\left|\phi_{t} * f_{2}(x)\right| \leq C$ on $\mathbf{R}^{n}$, we have

$$
\int_{B(0,2 R)} \Phi_{p(\cdot), q(\cdot)}\left(x,\left|\phi_{t} * f_{2}(x)\right|\right) d x \leq C .
$$

Further, noting that $\phi_{t} * f_{2}^{\prime}=0$ outside $B(0,2 R)$, we find

$$
\int_{\mathbf{R}^{n}} \Phi_{p(\cdot), q(\cdot)}\left(x,\left|\phi_{t} * f_{2}^{\prime}(x)\right|\right) d x \leq C .
$$

Therefore it suffices to prove

$$
\int_{\mathbf{R}^{n} \backslash B(0,2 R)} \Phi_{p(\cdot), q(\cdot)}\left(x,\left|\phi_{t} * f_{2}^{\prime \prime}(x)\right|\right) d x \leq C .
$$

Thus, in the rest of the proof, we may assume that $0 \leq f<1$ on $\mathbf{R}^{n}$ and $f=0$ on $B(0, R)$. Note that

$$
\int_{B(0,|x| / 2)} \phi_{t}(x-y) f(y) d y=0
$$


for $|x|>2 R$. Hence, applying Lemma 3.3, we have

$$
\left|\phi_{t} * f(x)\right|^{p(x)} \leq C\left(\left|\phi_{t}\right| * h(x)+|x|^{-A}\right)
$$

for $|x|>2 R$, where $h(y)=\Phi_{p(\cdot), q(\cdot)}(y, f(y))$. Thus the integration yields

$$
\int_{\mathbf{R}^{n} \backslash B(0,2 R)}\left|\phi_{t} * f(x)\right|^{p(x)} d x \leq C,
$$

which completes the proof.

We are now ready to prove Theorem 1.2.

Proof of Theorem 1.2. Given $\varepsilon>0$, choose a bounded function $g$ with compact support such that $\|f-g\|_{\Phi_{p(\cdot), q(\cdot)}, \mathbf{R}^{n}}<\varepsilon$. As in the proof of Theorem 1.1, using Theorem 3.4 this time, we have

$$
\left\|\phi_{t} * f-f\right\|_{\Phi_{p(\cdot), q(\cdot)}, \mathbf{R}^{n}} \leq C \varepsilon+\left\|\phi_{t} * g-g\right\|_{\Phi_{p(\cdot), q(\cdot)}, \mathbf{R}^{n}} .
$$

Obviously, $g \in L^{p_{0}}\left(\mathbf{R}^{n}\right)$. Hence by Lemma 3.1, $\phi_{t} * g \rightarrow g$ almost everywhere in $\mathbf{R}^{n}$. Since there is a compact set $S$ containing all the supports of $\phi_{t} * g$,

$$
\left\|\phi_{t} * g-g\right\|_{\Phi_{p(\cdot), q(\cdot)}, \mathbf{R}^{n}} \leq C^{\prime}\left\|\phi_{t} * g-g\right\|_{L^{p_{+}+1}, \mathbf{R}^{n}}
$$

with $C^{\prime}$ depending on $|S|$, and the Lebesgue convergence theorem implies $\| \phi_{t} * g-$ $g \|_{L^{p_{+}+1}, \mathbf{R}^{n}} \rightarrow 0$ as $t \rightarrow \infty$. Hence

$$
\limsup _{t \rightarrow 0}\left\|\phi_{t} * f-f\right\|_{\Phi_{p(\cdot), q(\cdot)}, \mathbf{R}^{n}} \leq C \varepsilon,
$$

which completes the proof.

Remark 3.5. In Theorem 1.2 (and in Theorem A), the condition $\phi \in L^{\left(p_{-}\right)^{\prime}}\left(\mathbf{R}^{n}\right)$ cannot be weakened to $\phi \in L^{q}\left(\mathbf{R}^{n}\right)$ for $1 \leq q<\left(p_{-}\right)^{\prime}$. In fact, for given $p_{1}>1$ and $1 \leq q<\left(p_{1}\right)^{\prime}$, we can find a smooth exponent $p(\cdot)$ on $\mathbf{R}^{n}$ such that $p_{-}=p_{1}$, $f \in L^{p(\cdot)}\left(\mathbf{R}^{n}\right)$ and $\phi \in L^{q}\left(\mathbf{R}^{n}\right)$ having compact support for which

$$
\|\phi * f\|_{L^{p(\cdot)}, \mathbf{R}^{n}}=\infty .
$$

For this, let $a \in \mathbf{R}^{n}$ be a fixed point with $|a|>1$ and let $p_{2}$ satisfy

$$
\frac{1}{\left(p_{1}\right)^{\prime}}+\frac{1}{p_{2}}<\frac{1}{q} \text {. }
$$

Then choose a smooth exponent $p(\cdot)$ on $\mathbf{R}^{n}$ such that

$$
p(x)=p_{1} \text { for } x \in B(0,1 / 2), \quad p(x)=p_{2} \text { for } x \in B(a, 1 / 2),
$$

$p_{-}=p_{1}$ and $p(x)=$ const. outside $B(0,|a|+1)$. Take

$$
\phi_{j}=j^{n / q} \chi_{B\left(a, j^{-1}\right)} \quad \text { and } \quad f_{j}=j^{n / p_{1}} \chi_{B\left(0, j^{-1}\right)}, \quad j=2,3, \ldots
$$

Then

$$
\left\|\phi_{j}\right\|_{L^{q}, \mathbf{R}^{n}}=C<\infty \quad \text { and } \quad\left\|f_{j}\right\|_{L^{p(\cdot), \mathbf{R}^{n}}}=\left\|f_{j}\right\|_{L^{p_{1}, B(0,1 / 2)}}=C<\infty .
$$

Note that if $x \in B\left(a, j^{-1}\right)$, then

$$
\phi_{j} * f_{j}(x)=j^{n / q+n / p_{1}}\left|B\left(a, j^{-1}\right) \cap B\left(x, j^{-1}\right)\right| \geq C j^{n / q+n / p_{1}} j^{-n},
$$


so that

$$
\begin{aligned}
\int_{\mathbf{R}^{n}}\left\{\phi_{j} * f_{j}(x)\right\}^{p(x)} d x & \geq \int_{B\left(a, j^{-1}\right)}\left\{\phi_{j} * f_{j}(x)\right\}^{p(x)} d x \\
& \geq C j^{p_{2}\left(n / q+n / p_{1}-n\right)} j^{-n} \\
& =C j^{p_{2} n\left(1 / q-1 /\left(p_{1}\right)^{\prime}-1 / p_{2}\right)}
\end{aligned}
$$

Now consider

$$
\phi=\sum_{j=2}^{\infty} j^{-2} \phi_{2^{j}} \quad \text { and } \quad f=\sum_{j=2}^{\infty} j^{-2} f_{2^{j}} .
$$

Then $\phi \in L^{q}\left(\mathbf{R}^{n}\right)$ and $f \in L^{p(\cdot)}\left(\mathbf{R}^{n}\right)$. On the other hand,

$$
\begin{aligned}
\int_{\mathbf{R}^{n}}\{\phi * f(x)\}^{p(x)} d x & \geq j^{-4} \int_{\mathbf{R}^{n}}\left\{\phi_{2^{j}} * f_{2^{j}}(x)\right\}^{p(x)} d x \\
& \geq C j^{-4} 2^{p^{2 n j}\left(1 / q-1 /\left(p_{1}\right)^{\prime}-1 / p_{2}\right)} \rightarrow \infty
\end{aligned}
$$

as $j \rightarrow \infty$. Hence, $\|\phi * f\|_{L^{p(\cdot)}, \mathbf{R}^{n}}=\infty$.

Remark 3.6. Cruz-Uribe and Fiorenza [1] gave an example showing that it can occur

$$
\limsup _{t \rightarrow 0}\left\|\phi_{t} * f\right\|_{L^{p(\cdot), \mathbf{R}}}=\infty
$$

for $f \in L^{p(\cdot)}(\mathbf{R})$ when $\phi$ does not have compact support.

By modifying their example, we can also find $p(\cdot)$ and $\phi \in L^{\left(p_{-}\right)^{\prime}}(\mathbf{R})$, whose support is not compact, such that

$$
\|\phi * f\|_{L^{p(\cdot), \mathbf{R}}} \leq C\|f\|_{L^{p(\cdot), \mathbf{R}}}
$$

does not hold, namely there exists $f_{N}(N=1,2, \ldots)$ such that $\left\|f_{N}\right\|_{L^{p(\cdot), \mathbf{R}}} \leq 1$ and

$$
\lim _{N \rightarrow \infty}\left\|\phi * f_{N}\right\|_{L^{p(\cdot), \mathbf{R}}}=\infty .
$$

For this purpose, choose $p_{1}>1, p_{2}>p_{1}$ and $a>1$ such that

$$
-p_{1} / p_{2}-a p_{1}+2>0
$$

and let $p(\cdot)$ be a smooth variable exponent on $\mathbf{R}$ such that

$$
p(x)=p_{1} \text { for } x \leq 0, \quad p(x)=p_{2} \text { for } x \geq 1
$$

and $p_{1} \leq p(x) \leq p_{2}$ for $0<x<1$. Set $\phi=\sum_{j=1}^{\infty} \chi_{j}$, where $\chi_{j}=\chi_{\left[-j,-j+j^{-a}\right)}$. Then

$$
\int_{\mathbf{R}} \phi(x)^{q} d x=\sum_{j=1}^{\infty} \int_{-j}^{-j+j^{-a}} \chi_{j}(x)^{q} d x=\sum_{j} j^{-a} \leq C(a)<\infty
$$

for any $q>0$. Further set $f_{N}=N^{-1 / p_{2}} \chi_{[1, N+1]}$. Note that for $1-j+j^{-a}<x<0$ and $j \leq N$

$$
\chi_{j} * f_{N}(x) \geq \int_{x+j-j^{-a}}^{x+j} \chi_{j}(x-y) f_{N}(y) d y=N^{-1 / p_{2}} j^{-a}
$$


so that

$$
\begin{aligned}
\int_{\mathbf{R}}\left\{\phi * f_{N}(x)\right\}^{p(x)} d x & \geq \int_{-\infty}^{0}\left\{\sum_{j=1}^{\infty} \chi_{j} * f_{N}(x)\right\}^{p_{1}} d x \\
& \geq \sum_{j=2}^{N} \int_{1-j-j-a}^{0}\left\{\chi_{j} * f_{N}(x)\right\}^{p_{1}} d x \\
& \geq N^{-p_{1} / p_{2}} \sum_{j=2}^{N} j^{-a p_{1}}\left(j-j^{-a}-1\right) \\
& \geq C N^{-p_{1} / p_{2}-a p_{1}+2} \rightarrow \infty \quad(N \rightarrow \infty) .
\end{aligned}
$$

\section{Young type inequalities}

Cruz-Uribe and Fiorenza [1] conjectured that Theorem A remains true if $\phi$ satisfies the additional condition

$$
|\phi(x-y)-\phi(x)| \leq \frac{|y|}{|x|^{n+1}} \quad \text { when }|x|>2|y| .
$$

Noting that this condition implies

$$
\sup _{x, z \in B\left(0,2^{j+1}\right) \backslash B\left(0,2^{j}\right)}|\phi(x)-\phi(z)| \leq C 2^{-n j},
$$

we see that $\lim _{|x| \rightarrow \infty} \phi(x)=0$ since $\phi \in L^{1}\left(\mathbf{R}^{n}\right)$ and

$$
|\phi(x)| \leq C|x|^{-n} .
$$

if $\phi$ satisfies (4.1). In this connection we show

Theorem 4.1. Let $p_{-}>1$. Suppose that $\phi \in L^{1}\left(\mathbf{R}^{n}\right) \cap L^{\left(p_{0}\right)^{\prime}}(B(0, R))$ and $\phi$ satisfies (4.2) for $|x| \geq R$. Then

$$
\|\phi * f\|_{\Phi_{p(\cdot), q(\cdot)}, \mathbf{R}^{n}} \leq C\left(\|\phi\|_{L^{1}, \mathbf{R}^{n}}+\|\phi\|_{L^{\left(p_{0}\right)^{\prime}, B(0, R)}}\right)\|f\|_{\Phi_{p(\cdot), q(\cdot)}, \mathbf{R}^{n}}
$$

for all $f \in L^{p(\cdot)}(\log L)^{q(\cdot)}\left(\mathbf{R}^{n}\right)$.

Remark 4.2. Theorem 4.1 does not imply an inequality

$$
\left\|\phi_{t} * f\right\|_{\Phi_{p(\cdot), q(\cdot)}, \mathbf{R}^{n}} \leq C\|f\|_{\Phi_{p(\cdot), q(\cdot)}, \mathbf{R}^{n}}
$$

with a constant $C$ independent of $t \in(0,1]$ even if $\phi$ satisfies (4.2) for all $x$, because $\left\{\left\|\phi_{t}\right\|_{L^{\left(p_{0}\right)^{\prime}, B(0, R)}}\right\}_{0<t \leq 1}$ is not bounded.

Proof of Theorem 4.1. Let $f$ be a nonnegative measurable function on $\mathbf{R}^{n}$ such

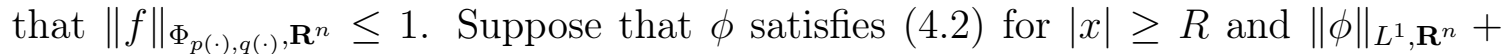
$\|\phi\|_{L^{\left(p_{0}\right)^{\prime}, B(0, R)}} \leq 1$. Decompose $\phi=\phi^{\prime}+\phi^{\prime \prime}$, where $\phi^{\prime}=\phi \chi_{B(0, R)}$. We first note by Theorem 1.2 that

$$
\left\|\phi^{\prime} * f\right\|_{\Phi_{p(\cdot), q(\cdot)}, \mathbf{R}^{n}} \leq C .
$$

Hence it suffices to show that

$$
\left\|\phi^{\prime \prime} * f\right\|_{\Phi_{p(\cdot), q(\cdot)}, \mathbf{R}^{n}} \leq C .
$$

For this purpose, write

$$
f=f \chi_{\left\{y \in \mathbf{R}^{n}: f(y) \geq 1\right\}}+f \chi_{\left\{y \in \mathbf{R}^{n}: f(y)<1\right\}}=f_{1}+f_{2},
$$


as before. Then we have by (4.2) and $(\Phi)$

$$
\begin{aligned}
\left|\phi^{\prime \prime} * f_{1}(x)\right| & \leq C \int_{\mathbf{R}^{n} \backslash B(x, R)}|x-y|^{-n} f_{1}(y) d y \\
& \leq C R^{-n} \int_{\mathbf{R}^{n}} f_{1}(y) d y \\
& \leq C R^{-n} \int_{\mathbf{R}^{n}} \Phi_{p(\cdot), q(\cdot)}(y, f(y)) d y \leq C .
\end{aligned}
$$

Noting that $\left|\phi^{\prime \prime} * f_{2}\right| \leq 1$, we obtain

$$
\int_{B(0, R)} \Phi_{p(\cdot), q(\cdot)}\left(x, \phi^{\prime \prime} * f(x)\right) d x \leq C .
$$

Next, let $h(y)=\Phi_{p(\cdot), q(\cdot)}(y, f(y))$. Then

$$
\left|\phi^{\prime \prime}\right| * h(x) \leq C R^{-n} \int_{\mathbf{R}^{n}} h(y) d y \leq C R^{-n} .
$$

If $x \in \mathbf{R}^{n} \backslash B(0, R)$, then we have by (4.2) and Lemma 3.3

$$
\begin{aligned}
\left|\phi^{\prime \prime} * f(x)\right| & \leq \int_{B(0,|x| / 2)}\left|\phi^{\prime \prime}(x-y)\right| f(y) d y+\int_{\mathbf{R}^{n} \backslash B(0,|x| / 2)}\left|\phi^{\prime \prime}(x-y)\right| f(y) d y \\
& \leq C\left\{|x|^{-n} \int_{B(x, 3|x| / 2)} f(y) d y+\left(\left|\phi^{\prime \prime}\right| * h(x)\right)^{1 / p(x)}+|x|^{-A / p(x)}\right\} \\
& \leq C\left\{M f(x)+\left(\left|\phi^{\prime \prime}\right| * h(x)\right)^{1 / p(x)}+|x|^{-A / p(x)}\right\}
\end{aligned}
$$

with $A>n$. Now it follows from Proposition 2.5 that

$$
\begin{aligned}
\int_{\mathbf{R}^{n} \backslash B(0, R)} \Phi_{p(\cdot), q(\cdot)}\left(x,\left|\phi^{\prime \prime} * f(x)\right|\right) d x \leq C & \left\{\int_{\mathbf{R}^{n} \backslash B(0, R)} \Phi_{p(\cdot), q(\cdot)}(x, M f(x)) d x\right. \\
& \left.+\int_{\mathbf{R}^{n}}|\phi| * h(x) d x+\int_{\mathbf{R}^{n} \backslash B(0, R)}|x|^{-A} d x\right\} \\
\leq C, &
\end{aligned}
$$

as required.

Theorem 4.3. Let $1-p_{-} / p_{+} \leq \theta<1,1<\tilde{p}<p_{-}$,

$$
\frac{1}{s}=1-\frac{\theta}{\tilde{p}} \text { and } \quad \frac{1}{r(x)}=\frac{1-\theta}{p(x)} .
$$

Take $\nu=p_{-} / \tilde{p}$, if $t^{-p_{-} / \tilde{p}} \Phi_{p(\cdot) / \tilde{p}, q(\cdot)}(x, t)$ is uniformly almost increasing in $t$; otherwise choose $1 \leq \nu<p_{-} / \tilde{p}$. Suppose that $\phi \in L^{1}\left(\mathbf{R}^{n}\right) \cap L^{s}\left(\mathbf{R}^{n}\right) \cap L^{s \nu^{\prime}}(B(0, R))$ and $\phi$ satisfies

$$
|\phi(x)| \leq C|x|^{-n / s}
$$

for $|x| \geq R$. Then

$$
\|\phi * f\|_{\Phi_{r(\cdot), q(\cdot)}, \mathbf{R}^{n}} \leq C\left(\|\phi\|_{L^{1}, \mathbf{R}^{n}}+\|\phi\|_{L^{s}, \mathbf{R}^{n}}+\|\phi\|_{L^{s \nu^{\prime}, B(0, R)}}\right)\|f\|_{\Phi_{p(\cdot), q(\cdot)}, \mathbf{R}^{n}}
$$

for all $f \in L^{p(\cdot)}(\log L)^{q(\cdot)}\left(\mathbf{R}^{n}\right)$. 
Proof. Suppose that $\|\phi\|_{L^{1}, \mathbf{R}^{n}}+\|\phi\|_{L^{s}, \mathbf{R}^{n}}+\|\phi\|_{L^{s \nu^{\prime}}, B(0, R)} \leq 1$ and $\phi$ satisfies

$$
|\phi(x)| \leq C|x|^{-n / s}
$$

for $|x| \geq R$. Let $f$ be a nonnegative measurable function on $\mathbf{R}^{n}$ such that $\|f\|_{\Phi_{p(\cdot), q(\cdot)}, \mathbf{R}^{n}}$ $\leq 1$, and decompose

$$
f=f_{1}+f_{2},
$$

where $f_{1}=f \chi_{\left\{x \in \mathbf{R}^{n}: f(x) \geq 1\right\}}$. Let

$$
\frac{1}{r}=\frac{1-\theta}{p_{-}} \text {and } \quad \frac{1}{s_{1}}=1+\frac{1}{r}-\frac{1}{p_{+}} .
$$

By our assumption, $s_{1} \geq 1$. It follows from Young's inequality for convolution that

$$
\left\|\phi * f_{2}\right\|_{L^{r}, \mathbf{R}^{n}} \leq\|\phi\|_{L^{s_{1}, \mathbf{R}^{n}}}\left\|f_{2}\right\|_{L^{p_{1}, \mathbf{R}^{n}}} .
$$

Here note that $1 \leq s_{1}<s$, so that $\|\phi\|_{L^{s_{1}, \mathbf{R}^{n}}} \leq\|\phi\|_{L^{1}, \mathbf{R}^{n}}+\|\phi\|_{L^{s}, \mathbf{R}^{n}} \leq 1$. Since $0 \leq f_{2}<1,\left\|f_{2}\right\|_{L^{p^{+}, \mathbf{R}^{n}}} \leq C\|f\|_{\Phi_{p(\cdot), q(\cdot)}, \mathbf{R}^{n}} \leq C$. Thus, noting that $\left|\phi * f_{2}\right| \leq 1$ and

$$
\frac{1}{r(x)}-\frac{1}{r}=\frac{1-\theta}{p(x)}-\frac{1-\theta}{p_{-}} \leq 0,
$$

we see that

$$
\left\|\phi * f_{2}\right\|_{\Phi_{r(\cdot), q(\cdot)}, \mathbf{R}^{n}} \leq C\left\|\phi * f_{2}\right\|_{L^{r}, \mathbf{R}^{n}} \leq C .
$$

On the other hand, we have by Hölder's inequality

$$
\begin{aligned}
\left|\phi * f_{1}(x)\right| \leq & \left(\int_{\mathbf{R}^{n}}|\phi(x-y)|^{s} f_{1}(y)^{\tilde{p}} d y\right)^{(1-\theta) / \tilde{p}}\left(\int_{\mathbf{R}^{n}}|\phi(x-y)|^{s} d y\right)^{1-1 / \tilde{p}} \\
& \cdot\left(\int_{\mathbf{R}^{n}}\left|f_{1}(y)\right|^{\tilde{p}} d y\right)^{\theta / \tilde{p}} \\
\leq & C\left(|\phi|^{s} * f_{1}^{\tilde{p}}(x)\right)^{(1-\theta) / \tilde{p}} .
\end{aligned}
$$

Noting that $|\phi|^{s} \in L^{1}\left(\mathbf{R}^{n}\right) \cap L^{\nu^{\prime}}(B(0, R)),|\phi|^{s}$ satisfies (4.2) for $|x| \geq R$ and $\left\|f_{1}^{\tilde{p}}\right\|_{\Phi_{p(\cdot) / \tilde{p}, q(\cdot)}, \mathbf{R}^{n}} \leq C$, we find by Theorem 4.1

$$
\left\|\phi^{s} * f_{1}^{\tilde{p}}\right\|_{\Phi_{p(\cdot) / \tilde{p}, q(\cdot)}, \mathbf{R}^{n}} \leq C .
$$

Since (4.4) implies

$$
\Phi_{r(\cdot), q(\cdot)}\left(x, \phi * f_{1}(x)\right) \leq C \Phi_{p(\cdot) / \tilde{p}, q(\cdot)}\left(x,|\phi|^{s} * f_{1}^{p_{1}}(x)\right),
$$

it follows that

$$
\left\|\phi * f_{1}\right\|_{\Phi_{r(\cdot), q(\cdot)}, \mathbf{R}^{n}} \leq C .
$$

Thus, together with (4.3), we obtain

$$
\|\phi * f\|_{\Phi_{r(\cdot), q(\cdot)}, \mathbf{R}^{n}} \leq C,
$$

as required.

Remark 4.4. Cruz-Uribe and Fiorenza [1] conjectured that Theorem A remains true if $\phi$ satisfies the additional condition (4.1).

If $p_{-}>1$, this conjecture was shown to be true by Cruz-Uribe, Fiorenza, Martell and Pérez in [3], using an extrapolation theorem ([3, Theorem 1.3 or Corollary 1.11]). Using our Proposition 2.5, we can prove the following extension of [3, Theorem 1.3]: 
Proposition 4.5. Let $\mathscr{F}$ be a family of ordered pairs $(f, g)$ of nonnegative measurable functions on $\mathbf{R}^{n}$. Suppose that for some $0<p_{0}<p^{-}$,

$$
\int_{\mathbf{R}^{n}} f(x)^{p_{0}} w(x) d x \leq C_{0} \int_{\mathbf{R}^{n}} g(x)^{p_{0}} w(x) d x
$$

for all $(f, g) \in \mathscr{F}$ and for all $A_{1}$-weights $w$, where $C_{0}$ depends only on $p_{0}$ and the $A_{1}$-constant of $w$. Then

$$
\|f\|_{\Phi_{p(\cdot), q(\cdot)}, \mathbf{R}^{n}} \leq C\|g\|_{\Phi_{p(\cdot), q(\cdot)}, \mathbf{R}^{n}}
$$

for all $(f, g) \in \mathscr{F}$ such that $g \in L^{p(\cdot)}(\log L)^{q(\cdot)}\left(\mathbf{R}^{n}\right)$.

Then, as in [3, p. 249], we can prove:

Theorem 4.6. Assume that $p_{-}>1$. If $\phi$ is an integrable function on $\mathbf{R}^{n}$ satisfying (4.1), then

$$
\left\|\phi_{t} * f\right\|_{\Phi_{p(\cdot), q(\cdot)}, \mathbf{R}^{n}} \leq C\|f\|_{\Phi_{p(\cdot), q(\cdot)}, \mathbf{R}^{n}}
$$

for all $t>0$ and $f \in L^{p(\cdot)}(\log L)^{q(\cdot)}\left(\mathbf{R}^{n}\right)$. If, in addition, $\int \phi(x) d x=1$, then

$$
\lim _{t \rightarrow 0}\left\|\phi_{t} * f-f\right\|_{\Phi_{p(\cdot), q(\cdot)}, \mathbf{R}^{n}=0 .}
$$

\section{Appendix}

For $p \geq 1, q \in \mathbf{R}$ and $c \geq e$, we consider the function

$$
\Phi(t)=\Phi(p, q, c ; t)=t^{p}(\log (c+t))^{q}, \quad t \in[0, \infty) .
$$

In this appendix, we give a proof of the following elementary result:

Theorem 5.1. Let $X$ be a non-empty set and let $p(\cdot)$ and $q(\cdot)$ be real valued functions on $X$ such that $1 \leq p(x) \leq p_{0}<\infty$ for all $x \in X$. Then, the following (1) and (2) are equivalent to each other:

(1) There exists $c_{0} \geq e$ such that $\Phi\left(p(x), q(x), c_{0} ; \cdot\right)$ is convex on $[0, \infty)$ for every $x \in X$;

(2) There exists $K>0$ such that $K(p(x)-1)+q(x) \geq 0$ for all $x \in X$.

This theorem may be well known; however, the authors fail to find any literature containing this result.

This theorem is a corollary to the following

Proposition 5.2. (1) If

$$
(1+\log c)(p-1)+q \geq 0,
$$

then $\Phi$ is convex on $[0, \infty)$.

(2) Given $p_{0}>1$ and $c \geq e$, there exists $K=K\left(p_{0}, c\right)>0$ such that $\Phi$ is not convex on $[0, \infty)$ whenever $1 \leq p \leq p_{0}$ and $q<-K(p-1)$.

Proof. By elementary calculation we have

$$
\Phi^{\prime \prime}(t)=t^{p-2}(c+t)^{-2}(\log (c+t))^{q-2} G(t)
$$

with

$G(t)=p(p-1)(c+t)^{2}(\log (c+t))^{2}+2 p q t(c+t) \log (c+t)-q t^{2} \log (c+t)+q(q-1) t^{2}$ for $t>0$. $\Phi(t)$ is convex on $[0, \infty)$ if and only if $G(t) \geq 0$ for all $t \in(0, \infty)$. 
(1) If $q \geq 0$, then

$$
G(t) \geq q t(2 p(c+t)-t) \log (c+t)-q t^{2} \geq q t(2 p c+2(p-1) t) \geq 0
$$

for all $t \in(0, \infty)$, so that $\Phi$ is convex on $[0, \infty)$.

If $-(1+\log c)(p-1) \leq q<0$, then

$$
\begin{aligned}
G(t)= & p\left\{\sqrt{p-1}(c+t) \log (c+t)+\frac{q}{\sqrt{p-1}} t\right\}^{2} \\
& -\frac{p q^{2}}{p-1} t^{2}-q t^{2} \log (c+t)+q(q-1) t^{2} \\
\geq & (-q) t^{2}\left(\frac{p q}{p-1}+\log c-(q-1)\right) \\
= & (-q) t^{2}\left(\frac{q}{p-1}+\log c+1\right) \geq 0
\end{aligned}
$$

for all $t \in(0, \infty)$, so that $\Phi$ is convex on $[0, \infty)$.

(2) If $p=1$ and $q<0$, then

$$
G(t)=q t((t+2 c) \log (c+t)+(q-1) t) \rightarrow-\infty
$$

as $t \rightarrow \infty$. Hence $\Phi$ is not convex on $[0, \infty)$.

Next, let $1<p \leq p_{0}$ and $q=-k(p-1)$ with $k>0$. Then

$$
\begin{aligned}
\frac{G(t)}{p-1} & =p((c+t) \log (c+t)-k t)^{2}+k(\log (c+t)-k+1) t^{2} \\
& \leq p_{0}((c+t) \log (c+t)-k t)^{2}+k(\log (c+t)-k+1) t^{2} .
\end{aligned}
$$

Let $\lambda=1-1 /\left(2 p_{0}\right)$. Then $0<\lambda<1$. If $k>(\log c) / \lambda$, there is (unique) $t_{k}>0$ such that $\log \left(c+t_{k}\right)=\lambda k$. Note that $t_{k} / k \rightarrow \infty$ as $k \rightarrow \infty$. We have

$$
\begin{aligned}
\frac{G\left(t_{k}\right)}{p-1} & \leq p_{0}\left(\left(c+t_{k}\right) \lambda k-k t_{k}\right)^{2}+k(\lambda k-k+1) t_{k}^{2} \\
& =k t_{k}^{2}\left\{\left(p_{0}(1-\lambda)-1\right)(1-\lambda) k+1-2 p_{0} c \lambda(1-\lambda) \frac{k}{t_{k}}+p_{0} c^{2} \lambda^{2} \frac{k}{t_{k}^{2}}\right\} .
\end{aligned}
$$

Since $p_{0}(1-\lambda)-1=-1 / 2$, it follows that there is $K=K\left(c, p_{0}\right)>(\log c) / \lambda$ such that $G\left(t_{k}\right)<0$ whenever $k \geq K$. Hence $\Phi$ is not convex if $1<p \leq p_{0}$ and $q \leq-K(p-1)$.

Acknowledgement. The authors are grateful to the referee for his/her valuable comments.

\section{References}

[1] Cruz-Uribe, D. and A. Fiorenza: Approximate identities in variable $L^{p}$ spaces. - Math. Nachr. 280, 2007, 256-270.

[2] Cruz-Uribe, D. and A. Fiorenza: $L \log L$ results for the maximal operator in variable $L^{p}$ spaces. - Trans. Amer. Math. Soc. 361, 2009, 2631-2647.

[3] Cruz-Uribe, D., A. Fiorenza, J. M. Martell, and C. PÉrez: The boundedness of classical operators on variable $L^{p}$ spaces. - Ann. Acad. Sci. Fenn. Math. 31, 2006, 239-264. 
[4] Cruz-Uribe, D., A. Fiorenza, and C. J. Neugebauer: The maximal function on variable $L^{p}$ spaces. - Ann. Acad. Sci. Fenn. Math. 28, 2003, 223-238; Ann. Acad. Sci. Fenn. Math. 29, 2004, 247-249.

[5] Diening, L.: Maximal function on Musielak-Orlicz spaces. - Bull. Sci. Math. 129, 657-700.

[6] Diening, L., P. Harjulehto, P. Hästö, Y. Mizuta, and T. Shimomura: Maximal functions in variable exponent spaces: limiting cases of the exponent. - Ann. Acad. Sci. Fenn. Math. $34: 2,2009,503-522$.

[7] KováčIK, O., and J. RÁkosník: On spaces $L^{p(x)}$ and $W^{k, p(x)}$. - Czechoslovak Math. J. 41, 1991, 592-618.

[8] Mizuta, Y., T. Ohno, and T. Shimomura: Sobolev's inequalities and vanishing integrability for Riesz potentials of functions in $L^{p(\cdot)}(\log L)^{q(\cdot)}$. - J. Math. Anal. Appl. 345, 2008, 70-85.

[9] Musielak, J.: Orlicz spaces and modular spaces. - Lecture Notes in Math. 1034, SpringerVerlag, Berlin, 1983.

[10] OrLicz, W.: Über konjugierte Exponentenfolgen. - Studia Math. 3, 1931, 200-211.

[11] Stein, E. M.: Singular integrals and differentiability properties of functions. - Princeton Univ. Press, Princeton, 1970.

[12] Zo, F.: A note on approximation of the identity. - Studia Math. 55, 1976, 111-122.

Received 25 June 2009 